\title{
The association between serum adhesion molecules and outcome in acute spontaneous intracerebral hemorrhage
}

Hung-Chen Wang ${ }^{1,2}$, Wei-Che Lin ${ }^{3}$, Yu-Jun Lin ${ }^{1,4}$, Cheng-Shyuan Rau', Tsung-Han Lee ${ }^{1}$, Wen-Neng Chang ${ }^{5}$, Nai-Wen Tsai ${ }^{5}$, Ben-Chung Cheng ${ }^{4,6}$, Chia-Te Kung ${ }^{7}$ and Cheng-Hsien Lu, ${ }^{4,5^{*}}$

\begin{abstract}
Introduction: Serum concentrations of adhesion molecules may be connected to the pathogenesis of secondary brain injury after spontaneous intracerebral hemorrhage $(\mathrm{ICH})$. This study posits the hypothesis that levels of adhesion molecules substantially increase after $\mathrm{ICH}$ and are decreased thereafter, and that they can predict treatment outcomes.

Methods: Two hundred and thirty-nine blood samples were collected from 60 consecutive patients admitted within 24 hours after onset of spontaneous $\mathrm{ICH}$ and 60 blood samples were collected from 60 volunteers. Additional samples were obtained on Days 4, 7, 10, and 14 after onset of ICH regardless of clinical deterioration.

Results: Upon discharge, the therapeutic outcomes of the 60 spontaneous $\mathrm{ICH}$ cases based on the modified Rankin Disability Scale (mMRS) showed that 17 had no disability while 8.3\% developed delayed cerebral infarction (DCl). Statistical analysis of adhesion molecules between patient groups with good outcome (mMRS $=0$ or 1$)$ and poor outcome (mMRS $\geq 2$ ) revealed significant differences in diabetes mellitus $(P=0.049)$, hyperlipidemia $(P=0.012)$, mentality change $(P=0.043), \mathrm{ICH}$ volume and intraventricular hemorrhage on admission ( $P=0.036$ and 0.006 , respectively), Glasgow Coma Scale (GCS) on admission $(P \leq 0.001)$, neuro-surgical intervention $(P=0.003)$, and sEselectin and soluble intercellular cell adhesion-molecule-1 (sICAM-1) levels on admission ( $P=0.036$ and 0.019 , respectively). Multiple logistic regression analysis of these significant variables showed that GCS on admission, hyperlipidemia, and sICAM-1 ( $P=0.039,0.042$, and 0.022 , respectively) were independently associated with outcome of acute spontaneous ICH.
\end{abstract}

Conclusion: Increased sICAM-1 and sE-selectin levels may imply poor therapeutic outcomes for the treatment of spontaneous ICH during hospitalization. These early inflammatory responses may cause whole-brain injury immediately after spontaneous $\mathrm{ICH}$ and offer a potential therapeutic target for such patients. The importance of these findings is that they offer a potential therapeutic target for patients with spontaneous ICH.

\section{Introduction}

Spontaneous intracerebral hemorrhage ( $\mathrm{ICH})$ is a fatal stroke subtype that accounts for $10 \%$ to $15 \%$ of all strokes. Its morbidity and mortality rates are among the highest, leaving individuals who survive with lasting disabilities [1-3]. Although $\mathrm{ICH}$ is a relatively common

\footnotetext{
* Correspondence: chlu99@ms44.url.com.tw

${ }^{4}$ Department of Biological Science, 70 Lienhai Road, National Sun Yat-Sen University, Kaohsiung, 80424, Taiwan

Full list of author information is available at the end of the article
}

disease, the patho-physiologic mechanisms responsible for the outcome are poorly understood.

The inflammatory response in ICH is characterized by activation of local immune cells like microglial cells. Acute inflammation is observed within 4 hours of $\mathrm{ICH}$ in animal models [4] and this local inflammatory reaction is partly responsible for brain damage after injury. Specifically, blood-derived leukocytes are the primary sources of this damage, and infiltration of systemic immune cells results in enhanced disruption of the blood-brain barrier (BBB), leading to increased cerebral 
edema and subsequent deterioration of neuro-behavioral function [5-7].

The injured endothelium promotes inflammation via upregulation of adhesion molecules such as soluble intercellular cell adhesion molecule-1 (sICAM-1), soluble endothelial selectin (sE-selectin), and soluble platelet selectin (sP-selectin) that bind to circulating leukocytes and facilitate their migration into the cerebral ischemic region [8]. Experimental and clinical data demonstrate significant elevation of blood markers of endothelial cells, platelet activation, and inflammatory cell adhesion molecules in acute ischemic stroke [9-11]. Adhesion molecules sICAM-1 and soluble vascular cell adhesion molecule-1 (sVCAM-1) are pro-inflammatory parameters for the activation of the immune system [12]. Their physiologic role is the regulation of cell-to-cell contacts [13]. Recruitment of activated peripheral blood mononuclear cells across endothelial cells of the BBB seems to be an essential step in the initiation of brain inflammation [14]. This step of immune cell entry into the brain tissue is regulated by adhesion molecules and leads to a complex cascade of events $[13,15]$. Moreover, adhesion molecules play a patho-physiologic role in cerebrovascular diseases [11]. One study has demonstrated that SICAM-1 and sVCAM-1 levels in cerebrospinal fluid (CSF) and serum of patients with acute subarachnoid hemorrhage are elevated compared with those of healthy controls [16]. Another study has found that highly elevated levels of sICAM-1 and sVCAM-1 in ventricular CSF are associated with lethal outcome after $\mathrm{ICH}$ [17]. This study investigated the relationship of serial inflammatory cell adhesion molecules and neuroimaging findings and clinical outcome after acute spontaneous ICH.

\section{Materials and methods \\ Patients}

This study on the time course of serum adhesion molecule levels in patients with spontaneous ICH was a post hoc analysis of prospectively collected data and was conducted over a period of 18 months (August 2009 to January 2011). During this period, 60 adult patients (at least 20 years old) were admitted within 24 hours after onset of spontaneous ICH to Kaohsiung Chang Gung Memorial Hospital, which is a 2,482-bed acute-care teaching hospital in southern Taiwan and which provides both primary and tertiary referral care.

The diagnosis of spontaneous ICH was confirmed by patient history, brain computed tomography (CT) scans, or brain magnetic resonance imaging (MRI) or a combination thereof. Patients were excluded if they had (a) imminent death, (b) diffuse atherosclerotic changes on intra- and extra-cranial vessels with or without evidence of old cerebral infarct, (c) central nervous infection during hospitalization, or (d) major systemic disease like end-stage renal disease, liver cirrhosis, or congestive heart failure.

All of the patients or their representatives provided written informed consent. The study was approved by the ethics committee of the Chang Gung Memorial Hospital institutional review board. Patients were under continuous observation and monitored for Glasgow Coma Scale (GCS) score, electrocardiogram, blood pressure, pulse rate, temperature, fluid balance, and laboratory parameters at regular intervals. Outcome was assessed upon discharge by using the modified Rankin Disability Scale (mMRS) [18]. Good outcome was defined as an mMRS score of less than 1, whereas poor outcome was an mMRS score of at least 2 or death.

\section{Data collection}

All of the patients underwent brain CT scan soon after arrival at the emergency room as well as serial follow-up brain CT every week during hospitalization. Emergency brain CT scan or MRI was performed if there was clinical deterioration (for example, acute-onset focal neurologic deficits, seizures, status epilepticus, and progressively disturbed consciousness) and as postneuro-surgical procedures. All of the patients received follow-up brain MRI to identify further cerebral lesions between days 10 and 14 after ICH or before discharge. Cerebral infarction was defined according to the criteria of the World Health Organization [19]. The principal investigator reviewed all available initial and follow-up CT scans and MRIs for the presence of ischemic lesions, and a second observer reviewed cases of equivocal findings. Neither one was aware of the laboratory results at the time of clinical and radiologic assessments.

\section{Blood sampling and laboratory investigations}

Two hundred thirty-nine blood samples from 60 patients were taken within 24 hours after onset of $\mathrm{ICH}$. The additional samples were obtained on days 4, 7, 10, and 14 after onset of ICH regardless of clinical deterioration. Sixty samples from 60 volunteers were also obtained. Blood samples were collected by venipuncture into Vacutainer SST tubes (BD, Franklin Lakes, NJ, USA). Blood was allowed to clot at room temperature for a minimum of 30 minutes. The clot was then removed by centrifugation at 3,000 rpm for 10 minutes at $4^{\circ} \mathrm{C}$. All serum samples were collected after centrifugation, isolated, and immediately stored at $-80^{\circ} \mathrm{C}$ in multiple aliquots.

Serum sICAM-1, sVCAM-1, sE-selectin, soluble leukocyte selectin (sL-selectin), and sP-selectin levels were assessed with commercially available enzyme-linked immunosorbent assays ( $\&$ D Systems, Minneapolis, MN, USA). In the assay, standards, controls, and 
unknown samples were incubated in micro-titration wells coated with marked (that is, anti-ICAM-1, VCAM1, P-selectin, E-selectin, and L-selectin) antibodies. After incubation and washing, the wells were treated with another anti-antigen detection antibody labeled with enzyme horseradish peroxidase.

After a second incubation and washing step, the wells were incubated with substrate tetramethylbenzidine. An acidic stopping solution was then added, and the degree of enzymatic turnover of the substrate was determined by a dual-wavelength absorbance measurement at 450 and $620 \mathrm{~nm}$. Absorbance was directly proportional to the concentration of antigens present. A set of standard antigen was used to plot a standard curve of absorbance versus antigen concentration from which the antigen concentrations of the unknowns were calculated.

\section{Data analysis}

Data were expressed as mean \pm standard derivation or as median (interquartile range, or IQR). Categorical variables were compared by using the chi-square test or Fisher exact test, where appropriate. Serum levels of adhesion molecules were logarithmically transformed to improve normality and compared by Student $t$ test. Repeated measures of analysis of variance were used to compare adhesion molecules at five different time points (days 1, 4, 7, 10, and 14), and Scheffé's multiple comparison was used to analyze the intra-individual course of parameters over time and to compare parameters of two different groups (good and poor outcome).

Correlation analysis by Spearman ranking test was used to explore the relationship between age, GCS score on admission, and score on the mMRS upon discharge and variables like soluble intercellular adhesion molecule on admission.

Stepwise logistic regression was used to evaluate the relationship between significant variables and therapeutic outcomes, and adjustments were made for other potential confounding factors. Variables with zero cell count in a two-by-two table were eliminated from logistic analysis, and only variables with strong association with poor outcome $(P<0.05)$ were included in the final model. Lastly, receiver operating characteristic (ROC) curves were generated for soluble adhesion molecule levels on admission. The areas under the ROC curves were calculated for each parameter and compared. All of the statistical analyses were conducted with the SAS software package, version 9.1 (2002; SAS Institute Inc., Cary, NC, USA).

\section{Results}

\section{Baseline characteristics of the study patients}

The baseline characteristics of the 60 adults with spontaneous ICH and 60 sex- and age-matched volunteers are listed in Table 1. The 60 patients with spontaneous ICH consisted of 42 men (age range of 36 to 75 years and mean age of 57.0 years) and 18 women (age range of 41 to 74 years and mean age of 59.9 years). Nineteen (31.7\%) had lobar ICH, and 41 (68.3\%) had deep ICH. Of the lobar hemorrhages, four were parietal, three frontal, five temporal, and seven occipital. The deep locations were the basal ganglia (26 patients) and thalamus (15 patients). Nineteen (31.7\%) had intraventricular hemorrhage and nine (10\%) had acute hydrocephalus at presentation. The median intracerebral hematoma was 10.0 (IQR of 5.7 to 25.8) at presentation. Treatments of $\mathrm{ICH}$ included extra-ventricular drainage in five, $10 \mathrm{cra}-$ niotomy, and three craniectomy. Fifty-five (91.7\%) had one or more underlying diseases: 55 had hypertension, 10 diabetes mellitus, two asthma, seven chronic obstructive pulmonary disease, five atrial fibrillation, four coronary artery disease, and 19 hyperlipidemia. Thirty-four were smokers.

In patients with spontaneous $\mathrm{ICH}$, in comparison with the volunteer subjects, sICAM-1 and SVCAM-1 concentrations markedly increased at presentation (205.0 and $709.7 \mathrm{ng} / \mathrm{mL}$, respectively) whereas sL-selectin markedly decreased $(759.4 \mathrm{ng} / \mathrm{mL})$ (Table 1). The differences between volunteers and spontaneous ICH patients were statistically significant $(P=0.021, P=0.015$, and $P \leq 0.000$, respectively). The $\mathrm{SP}$-selectin and $\mathrm{sE}$-selectin concentrations did not show any statistically significant difference between the two groups at presentation: 85.1 versus 84.9 $\mathrm{ng} / \mathrm{mL}(P=0.841)$ and 39.4 versus $40.9 \mathrm{ng} / \mathrm{mL}(P=0.600)$.

\section{Effect of soluble intercellular adhesion molecule levels on spontaneous intracerebral hemorrhage}

Correlation analysis was used to test the influence of soluble intercellular adhesion molecule levels at presentation on age, GCS score, and ICH volume at presentation. The statistical results (correlation coefficient, $P$ value) revealed that age significantly correlated with levels of sICAM-1 $(r=-0.422, P=0.003)$, sL-selectin $(r$ $=-0.422, P=0.003)$, and sE-selectin $(r=-0.427, P=$ 0.002). GCS score significantly correlated with sVCAM1 level $(r=-0.304, P=0.034)$. ICH volume did not have any significant correlation with these soluble intercellular adhesion molecules, whereas GCS score and $\mathrm{ICH}$ volume at presentation had a significant negative correlation with the molecules $(r=-0.564, P \leq 0.001)$.

Sixteen (27\%) of 60 patients received neuro-surgical treatments: nine within 24 hours, four between 24 and 48 hours, and three within 4 days after ICH. When the effect of neuro-surgical intervention on SICAM-1 and sE-selectin levels in patients with $\mathrm{ICH}$ was compared, both had increased trends on days 1 and 4. However, only sICAM-1 level had a statistically significant difference on day $4(P=0.025)$. 
Table 1 Demographic data of patients and volunteer subjects at admission

\begin{tabular}{|c|c|c|c|}
\hline Parameter & $\begin{array}{l}\text { Volunteer subjects } \\
\quad(n=60)\end{array}$ & $\begin{array}{l}\text { Patients } \\
(\mathrm{n}=60)\end{array}$ & $P$ value \\
\hline Age in years, mean \pm standard deviation & $57.5 \pm 5.7$ & $57.9 \pm 10.1$ & 0.790 \\
\hline Men & 42 & 42 & 1.000 \\
\hline \multicolumn{4}{|l|}{ Underlying diseases } \\
\hline Hypertension & 19 & 55 & $\leq 0.001^{\mathrm{a}}$ \\
\hline Diabetes mellitus & 9 & 10 & 0.465 \\
\hline Asthma & 3 & 2 & 1.000 \\
\hline Chronic obstructive pulmonary disease & 7 & 7 & 0.778 \\
\hline Atrial fibrillation & 10 & 5 & 0.419 \\
\hline Coronary artery disease & 1 & 4 & 0.171 \\
\hline Hyperlipidermia & 8 & 19 & $0.004^{\mathrm{a}}$ \\
\hline Smoking & 17 & 34 & $0.000^{\mathrm{a}}$ \\
\hline \multicolumn{4}{|l|}{ Laboratory data at presentation, median (IQR) } \\
\hline White blood cell count, $\times 10^{3} / \mathrm{mL}$ & $5.6(4.85,7.80)$ & $8.45(6.13,10.53)$ & $0.001^{\mathrm{a}}$ \\
\hline Platelet count, $\times 10^{3} / \mathrm{mL}$ & $245.0(202.0,306.5)$ & $206.0(168.3,355.8)$ & $0.019^{\mathrm{a}}$ \\
\hline \multicolumn{4}{|l|}{ Brain image findings at presentation } \\
\hline Intracerebral hemorrhage volume in cubic millimeters, median (IQR) & - & $10.0(5.7,25.8)$ & - \\
\hline Intraventricular hematoma & - & 19 & - \\
\hline Hydrocephalus & - & 9 & - \\
\hline Craniotomy & - & 16 & - \\
\hline \multicolumn{4}{|c|}{ Serum adhesion molecule levels in nanograms per milliliter at presentation, median (IQR) } \\
\hline SL-selectin & $970.5(844.8,1045.1)$ & $759.4(649.7,880.4)$ & $\leq 0.001^{\mathrm{a}}$ \\
\hline sP-selectin & $85.1(78.4,92.5)$ & $84.9(59.2,97.8)$ & 0.841 \\
\hline sE-selectin & $39.4(31.9,44.6)$ & $40.9(30.5,54.1)$ & 0.600 \\
\hline sICAM-1 & $183.7(155.0,202.2)$ & $205.0(159.8,240.0)$ & $0.021^{\mathrm{a}}$ \\
\hline sVCAM-1 & $613.5(527.4,674.4)$ & $709.7(549.5,934.1)$ & $0.015^{\mathrm{a}}$ \\
\hline
\end{tabular}

Values in the 'Volunteer subjects' and 'Patients' columns are presented as number of patients unless indicated otherwise. ${ }^{a} \mathrm{p} \leqq 0.05$; IQR, interquartile range; $\mathrm{sE}-$ selectin, soluble endothelial selectin; sICAM-1, soluble intercellular cell adhesion molecule-1; sL-selectin, soluble leukocyte selectin; sP-selectin, soluble platelet selectin; sVCAM-1, soluble vascular cell adhesion molecule-1.

\section{Outcome and prognostic factors}

Upon discharge, the therapeutic outcomes of 60 spontaneous ICH cases as determined by mMRS score showed that 17 had no disability, eight had slight disability, 10 had moderated disability, and 25 had severe disability, including five who became vegetative. There were no mortalities. Five $(8.3 \%)$ of 60 patients had delayed cerebral infarction during the acute stage of spontaneous $\mathrm{ICH}$ : two in the basal ganglion and one each in the frontal, temporal, and parietal lobes. The median duration of delayed cerebral infarction was 4 days (IQR of 1.5 to 8.0 days).

The clinical features, neuro-imaging findings, and laboratory data between the two patient groups of good outcome (mMRS score of 0 or 1 ) and poor outcome (mMRS of at least 2) were compared, and statistical analysis revealed significant difference in diabetes mellitus $(P=0.049)$, hyperlipidemia $(P=0.012)$, mentality change $(P=0.043)$, ICH volume and intraventricular hemorrhage on admission $(P=0.036$ and 0.006 , respectively), GCS score on admission $(P \leq 0.001)$, neuro-surgical intervention $(P=0.003)$, and $\mathrm{sE}$-selectin and sICAM-1 levels on admission $(P=0.036$ and 0.019 , respectively). The median lengths of hospitalization were 15 days (IQR of 13 to 23 days) and 11 days (IQR of 7 to 13 days) for those with poor and good outcome, respectively $(P \leq 0.001)$ (Table 2$)$.

\section{Time course of soluble intercellular adhesion molecule levels}

Compared with volunteers, plasma sICAM-1 and sVCAM-1 levels were elevated significantly after onset of $\mathrm{ICH}$ and peaked on days 10 (sICAM-1) and 4 (sVCAM-1) and then slightly decreased thereafter (Figure 1a,b). Plasma sL-selectin levels decreased significantly after ICH onset and reached the lowest level on day 4 and then increased thereafter (Figure 1d). Plasma $\mathrm{sP}$-selectin levels decreased after onset of $\mathrm{ICH}$, then increased gradually thereafter, and had a significant difference on day 14 only (Figure 1c). With the volunteers, there was no significant difference in plasma sE-selectin levels, which increased after $\mathrm{ICH}$ onset, peaked on day 4, and decreased thereafter (Figure 1e).

When the therapeutic outcomes in the 60 spontaneous ICH cases as determined by mMRS score were 


\begin{tabular}{|c|c|c|c|c|}
\hline & $\begin{array}{l}\text { Poor outcome } \\
\qquad n=43\end{array}$ & $\begin{array}{l}\text { Good outcome } \\
\qquad \mathrm{n}=17\end{array}$ & $\begin{array}{l}\text { Odds } \\
\text { ratio }\end{array}$ & $P$ value \\
\hline \multicolumn{5}{|l|}{ Age in years, mean \pm standard deviation } \\
\hline Men & 31 & 11 & 1.409 & 0.755 \\
\hline \multicolumn{5}{|l|}{ Underlying diseases } \\
\hline Hypertension & 40 & 15 & 1.778 & 0.616 \\
\hline Diabetes mellitus & 10 & 0 & & $0.049^{\mathrm{a}}$ \\
\hline Asthma & 2 & 0 & & 1.000 \\
\hline Chronic obstructive pulmonary disease & 5 & 2 & 0.987 & 1.000 \\
\hline Atrial fibrillation & 3 & 2 & 0.563 & 0.616 \\
\hline Coronary artery disease & 3 & 1 & 1.200 & 1.000 \\
\hline Hyperlipidermia & 9 & 10 & 0.185 & $0.012^{a}$ \\
\hline Smoking & 25 & 9 & 1.235 & 0.777 \\
\hline \multicolumn{5}{|l|}{ Clinical feature at presentation } \\
\hline Headache & 19 & 7 & 1.131 & 1.000 \\
\hline Brief unconsciousness & 26 & 14 & 0.328 & 0.136 \\
\hline Mentality change & 24 & 4 & 4.105 & $0.043^{\mathrm{a}}$ \\
\hline Seizure & 2 & 0 & & 1.000 \\
\hline \multicolumn{5}{|l|}{ Laboratory data at presentation, median (IQR) } \\
\hline White blood cell count, $\times 10^{3} / \mathrm{mL}$ & $8.7(6.8,10.6)$ & $7.2(5.7,10.0)$ & & 0.144 \\
\hline Platelet count, $\times 10^{3} / \mathrm{mL}$ & $\begin{array}{l}206.0(160.0 \\
259.0)\end{array}$ & $\begin{array}{l}205.0(171.5 \\
218.5)\end{array}$ & & 0.512 \\
\hline \multicolumn{5}{|l|}{ Brain image findings at presentation } \\
\hline Intracerebral hemorrhage volume in cubic millimeters, median (IQR) & $12.0(7.0,34.0)$ & $7.2(2.0,17.5)$ & & $0.036^{a}$ \\
\hline Intraventricular hemorrhage & 18 & 1 & 11.52 & $0.006^{\mathrm{a}}$ \\
\hline Hydrocephalus & 9 & 0 & & 0.050 \\
\hline Glasgow Coma Scale score at presentation, median (IQR) & $14(9,15)$ & $15(15,15)$ & & $\leq 0.001^{a}$ \\
\hline Surgical intervention & 16 & 0 & & $0.003^{\mathrm{a}}$ \\
\hline Extraventricular drainage & 5 & 0 & & 0.309 \\
\hline Craniotomy & 10 & 0 & & $0.049^{\mathrm{a}}$ \\
\hline Craniectomy & 3 & 0 & & 0.511 \\
\hline \multicolumn{5}{|c|}{$\begin{array}{l}\text { Serum adhesion molecule levels in nanograms per milliliter at presentation, median } \\
\text { (IQR) }\end{array}$} \\
\hline SL-selectin & $\begin{array}{l}771.5(638.9 \\
922.4)\end{array}$ & $\begin{array}{l}675.6(641.5 \\
783.2)\end{array}$ & & 0.111 \\
\hline sP-selectin & $85.7(58.9,97.9)$ & $80.3(60.2,93.8)$ & & 0.3664 \\
\hline sE-selectin & $43.3(33.4,63.6)$ & $32.8(21.3,45.7)$ & & $0.036^{\mathrm{a}}$ \\
\hline SICAM-1 & $\begin{array}{l}208.2(174.4 \\
259.3)\end{array}$ & $\begin{array}{l}170.3(100.2 \\
228.3)\end{array}$ & & $0.019^{a}$ \\
\hline sVCAM-1 & $\begin{array}{l}709.7(559.1 \\
938.8)\end{array}$ & $\begin{array}{l}653.9(502.0 \\
947.0)\end{array}$ & & 0.353 \\
\hline Delayed cerebral infarction & 4 & 1 & 0.609 & 1.000 \\
\hline Days of intensive care unit stay, median (IQR) & $5(2.8,8.3)$ & $2(1.5,3.5)$ & & $\leq 0.001^{a}$ \\
\hline Days of hospitalization, median (IQR) & $15(12.8,22.3)$ & $11(7,13)$ & & $\leq 0.001^{a}$ \\
\hline
\end{tabular}

Values in the 'Poor outcome' and 'Good outcome' columns are presented as number of patients unless indicated otherwise. The following tests were used: Student $t$ test (for age), Mann-Whitney $U$ test (for laboratory data and soluble intercellular adhesion molecule at admission), and Fisher exact test (for gender and underlying diseases). ${ }^{a} \mathrm{p} \leqq 0.05 ; \mathrm{IQR}$, interquartile range; $\mathrm{sE}$-selectin, soluble endothelial selectin; sICAM-1, soluble intercellular cell adhesion molecule-1; sL-selectin, soluble leukocyte selectin; sP-selectin, soluble platelet selectin; sVCAM-1, soluble vascular cell adhesion molecule-1.

compared, the sICAM-1 and sE-selectin concentration levels increased significantly from days 1 to 4 in patients with poor outcome (Figure 2a,e). Otherwise, the levels of sVCAM-1, sP-selectin, and sL-selectin were not significantly different during the first 14 days (Figure 2b,c,d).
Multiple logistic regression analysis of significant variables, including diabetes mellitus $(P=0.049)$, hyperlipidemia $(P=0.012)$, mentality change $(P=0.043), \mathrm{ICH}$ volume and intraventricular hemorrhage on admission $(P=0.036$ and 0.006 , respectively), GCS score on admission $(P \leq 0.001)$, neuro-surgical intervention $(P=0.003)$, 


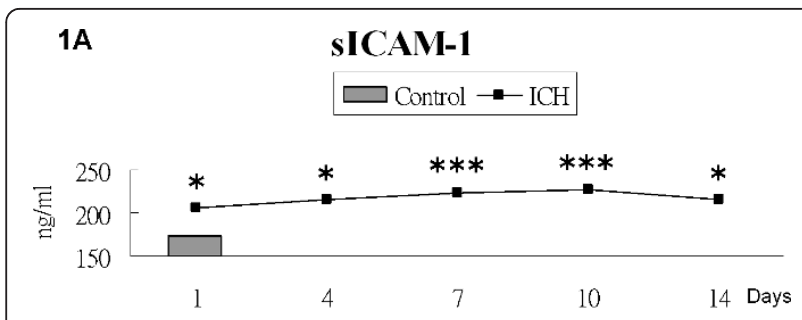

1B

sVCAM-1
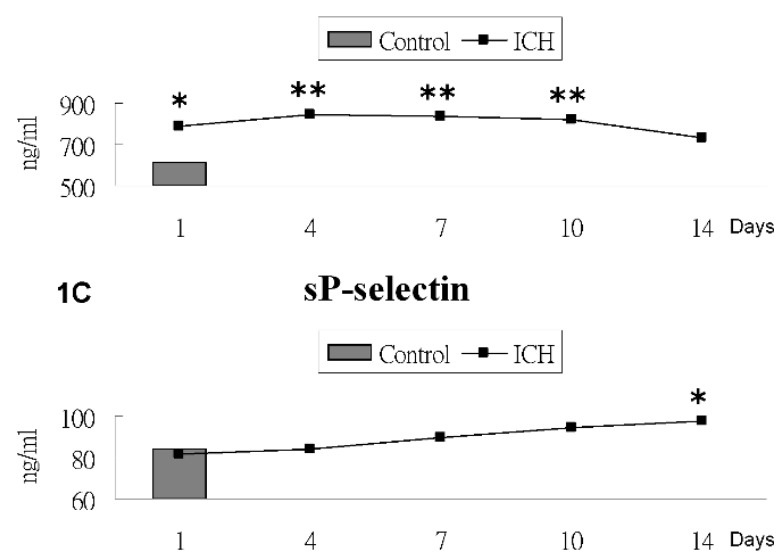

1D sL-selectin

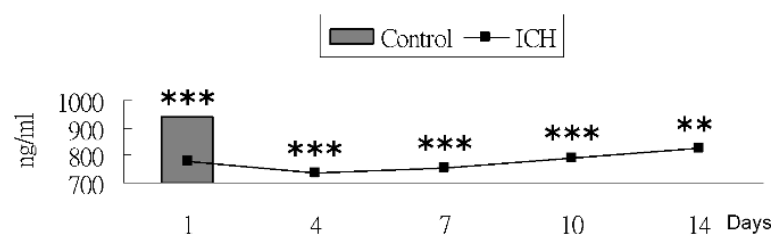

1E sE-selectin

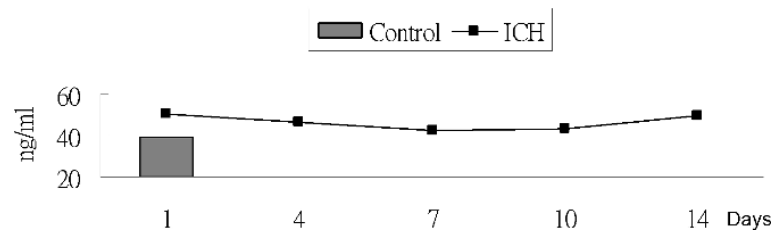

Figure 1 Serum levels of sICAM-1 (a), SVCAM-1 (b), sP-selectin (c), sL-selectin (d), and SE-selectin (e) on various days (days 1 , $4,7,10$, and 14) in patients with ICH and controls. ${ }^{*} P<0.05$, ${ }^{* *} P$ $<0.01,{ }^{* * *} P<0.001, \mathrm{ICH}$ patients versus controls. ICH, intracerebral hemorrhage; sE-selectin, soluble endothelial selectin; sICAM-1, soluble intercellular cell adhesion molecule-1; sL-selectin, soluble leukocyte selectin; sP-selectin, soluble platelet selectin; SVCAM-1, soluble vascular cell adhesion molecule-1.

and time course of sE-selectin and sICAM-1, showed that GCS score on admission, hyperlipidemia, and sICAM-1 $(P=0.039,0.042$, and 0.022 , respectively) were independently associated with outcome of acute spontaneous ICH. Furthermore, the ROC curves revealed that the cutoff point of sICAM-1 on admission was $194.61 \mathrm{ng} / \mathrm{mL}$ (sensitivity of $68.8 \%$, specificity of
$71.4 \%$, area of 0.717 , and $95 \%$ confidence interval of 0.553 to 0.880$)$.

\section{Discussion}

The present study examines the corrections between outcome and endothelial cell activation markers following spontaneous $\mathrm{ICH}$ and produces the following major findings. First, sICAM-1 and sVCAM-1 concentrations markedly increased in patients with spontaneous $\mathrm{ICH}$ at presentation (205.0 and $709.7 \mathrm{ng} / \mathrm{mL}$, respectively), whereas sL-selectin markedly decreased $(759.4 \mathrm{ng} / \mathrm{mL})$ (Table 1). Second, plasma concentrations of sICAM-1 and sVCAM-1 elevated significantly after ICH onset, peaked on day 10 (sICAM-1) and day 4 (sVCAM-1), and then slightly decreased thereafter (Figure 1a,b). Furthermore, plasma sL-selectin levels decreased significantly after onset of $\mathrm{ICH}$, reached its lowest level on day 4 , and then increased thereafter (Figure 1d). Third, when the therapeutic outcomes of the 60 spontaneous $\mathrm{ICH}$ cases as determined by mMRS score were compared, sICAM and sE-selectin concentration levels increased significantly from days 1 to 14 in patients with poor outcome (Figure 2a,e). Fourth, GCS score on admission, hyperlipidemia as the underlying disease, and sICAM-1 $(P=0.039,0.042$, and 0.022 , respectively $)$ were independently associated with outcome of acute spontaneous ICH.

\section{Adhesion molecules}

Several studies have shown that adhesion molecules play a patho-physiologic role in cerebrovascular diseases $[11,16,17,20]$. One study has demonstrated that sICAM1 and sVCAM-1 levels in CSF and sera of patients with acute subarachnoid hemorrhage are elevated compared with those of healthy controls [16]. Two studies by Kraus and colleagues $[17,20]$ have found that highly elevated levels of SICAM-1 and SVCAM-1 in ventricular CSF, but not in serum, are associated with lethal outcome after ICH. Only sICAM-1 and sVCAM-1 levels in CSF, not in serum, are significantly correlated with basal ganglia hemorrhage volume and edema volume. However, there is no significant correlation with outcome at day 8 or at 6 months later [20].

In the former study by Kraus and colleagues [17], patients were categorized into one of two groups (patients who survived and patients who died). In the former study by Kraus and colleagues [17],there were no normal subjects for comparison that may mask the true difference in adhesion molecules in different disease severities. Furthermore, the two studies have relatively small groups of patients ( $\mathrm{n}=10$ and 11 , respectively). The present study shows increased concentration levels of SICAM-1 and SVCAM-1 in patients with acute spontaneous ICH. This activation persists for at least 14 days 

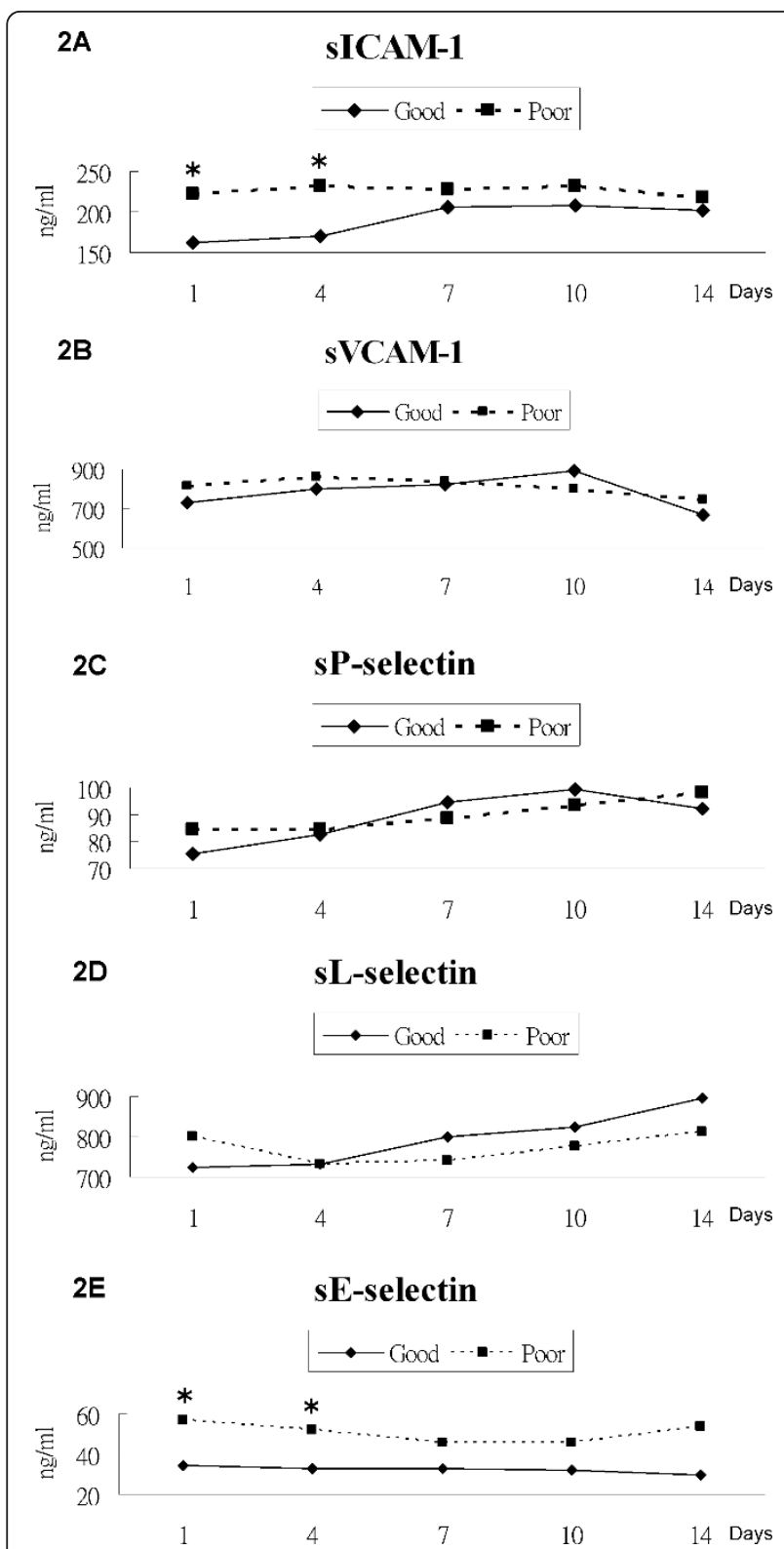

Figure 2 Serum levels of SICAM-1 (a), sVCAM-1 (b), sP-selectin (c), sL-selectin (d), and sE-selectin (e) on various days (days 1 , $4,7,10$, and 14 ) in patients with intracerebral hemorrhage. ${ }^{*} P$ $<0.05$, good outcome versus poor outcome. sE-selectin, soluble endothelial selectin; SICAM-1, soluble intercellular cell adhesion molecule-1; sL-selectin, soluble leukocyte selectin; sP-selectin, soluble platelet selectin; sVCAM-1, soluble vascular cell adhesion molecule-1.

after ICH onset and suggests that the activation of adhesion molecules, especially sICAM-1 and sVCAM- 1 , plays a patho-physiologic role in the acute phases after spontaneous $\mathrm{ICH}$.

The inflammatory response in ICH is characterized by the activation of local immune cells such as microglial cells. This local inflammatory reaction is partly responsible for damages to the brain after injury. Mounting evidence also suggests that systemic immune cells, specifically blood-derived leukocytes, are the primary sources of this damage [4]. In focal ischemic stroke models, systemic immune cell recruitment is mediated in part by the increase in adhesion molecule expression along the endothelial cell walls [8]. As a result, these systemic immune cells propagate local immune response by releasing pro-inflammatory cytokines at the site of injury, increasing cerebral edema, and worsening neuro-behavioral function $[5,9,10]$.

Previous studies on ICH indicate that brain edema increases progressively in the first 24 hours and remains elevated for several days because of an inflammatory response characterized by the infiltration of neutrophils and macrophages as well as the activation of microglia [6]. The cause of secondary brain injury and edema formation is uncertain. The results of the present study show that an inflammatory response occurs after $\mathrm{ICH}$ and that adhesion molecules, especially sICAM-1 and sE-selectin, may play an important role in the inflammatory response, contributing to secondary brain injury and worsened neuro-behavioral function.

\section{Delayed cerebral infarction}

Five $(8.3 \%)$ of 60 patients in the present study had delayed cerebral infarction during hospitalization, and this incidence is similar to that of a previous report [21]. However, these adhesion molecules are not correlated with the occurrence of delayed cerebral infarction, and patients who had delayed cerebral infarction do not account for the outcome in this study. Several mechanisms are implicated in the development of cerebral infarctions following spontaneous ICH. One is the massive release of blood and blood breakdown products into the CSF and subsequent inflammatory changes in the smooth muscle of the large cerebral arteries as well as released vasoactive substances involving inflammatory host reactions [22-24]. As the disease progresses, inflammatory changes result in endothelial dysfunction with consequences that include loss of cerebrovascular autoregulation and increased permeability of the $\mathrm{BBB}$. Decreased cerebral blood flow becomes apparent and leads to cerebral ischemia $[25,26]$. The possible reasons that delayed cerebral infarction does not significantly correlate with adhesion molecules in the present study are that the conditions of the ICH patient population are mild at admission: median GCS score of 14.5 (IQR of 10.3 to 15.0 ) and median $\mathrm{ICH}$ volume of $10.0 \mathrm{~mL}$ (IQR of 5.7 to $25.8 \mathrm{~mL}$ ). Therefore, the inflammatory condition in the patient population may be less severe and does not cause worse outcome.

Of the 60 patients enrolled in this study, 42 (70\%) were mild, eight (13.3\%) were moderate, and 10 (16.7\%) 
were severe in their initial GCS score. The hematoma volume was relatively small: the median volume of intracerebral hematoma was $10.0 \mathrm{~mL}$ (IQR of 5.7 to 25.8 $\mathrm{mL}$ ) at presentation. The small hematoma volume will cause less volume effect, and this may explain why the intracerebral hematoma volume is not an independent factor associated with the outcome.

In this study, underlying disease with hyperlipidemia is independently associated with outcome of acute spontaneous $\mathrm{ICH}$. At the moment of acute spontaneous $\mathrm{ICH}$, the patients were all being treated for hyperlipidemia with statin drugs. Previous studies reveal that pre-treatment with intense low-density lipoprotein cholesterolreducing drugs improves the clinical outcome in patients with ischemic and hemorrhagic stroke [27-29]. Previous reports demonstrate an induction of apoptosis, inflammation, and excitotoxic cascades after ICH [30]. There is also increasing evidence that statins have antiinflammatory and anti-thrombotic effects, aside from their cholesterol-lowering effect $[31,32]$.

In the patient population here, neuro-surgical intervention is significantly associated with poor outcome. It is reasonable to postulate that surgical intervention can affect the serum levels of various adhesion molecules. All of the neuro-surgical treatments were done within 4 days after ICH. However, only the level of sICAM-1 had a statistically significant difference on day $4(P=0.025)$ between patients with neuro-surgical intervention and those without it. Furthermore, when included in the multiple logistic regression analysis, neuro-surgical intervention is not an independent factor associated with outcome of acute spontaneous $\mathrm{ICH}$. Thus, neurosurgical intervention may affect the serum levels of various adhesion molecules but does not confound the major findings of the study.

This study has several limitations. First, patients who were deeply comatose or those with imminent death were excluded. There is uncertainty in assessing the expression of these adhesion molecules in critically ill and high-risk patients. Second, sICAM-1, sVCAM-1, sPselectin, sL-selectin, and sE-selectin are only part of the endothelium-leukocyte-platelet adherent molecules expressed after cerebrovascular diseases. Therefore, increased levels of these markers may not necessarily be reflected in the actual patho-physiologic function. Third, serial changes of adhesion molecules may be influenced by the expression of platelet adhesion molecules (CD62P, CD63, and CD40L), platelet-leukocyte interaction, and several drugs (for example, statins and calcium channel blockers), which are commonly used in patients with ICH. Lastly, the case numbers are small and the follow-up period is short. Large-scale studies with longer follow-up periods to evaluate the association between clinical outcome and the expression of adhesion molecules are warranted.

\section{Conclusions}

An increase in sICAM-1 and sE-selectin levels implies a danger of poor therapeutic outcome for the treatment of spontaneous ICH during hospitalization. These early inflammatory responses may cause whole-brain injury immediately after spontaneous ICH. The importance of these findings is that they offer a potential therapeutic target for such patients. Further large-scale studies are needed to elucidate the relevance of serum concentrations of adhesion molecules and secondary brain injury after spontaneous $\mathrm{ICH}$.

\section{Key messages}

- Increased levels of soluble intercellular cell adhesion molecule-1 (sICAM-1) and soluble endothelial selectin (sE-selectin) imply a danger of poor therapeutic outcome for the treatment of acute spontaneous intracerebral hemorrhage ( $\mathrm{ICH})$.

- Early inflammatory responses may cause wholebrain injury immediately after spontaneous ICH.

- These findings are important because they offer a potential therapeutic target for patients with spontaneous $\mathrm{ICH}$.

\section{Abbreviations}

BBB: blood-brain barrier; CSF: cerebrospinal fluid; CT: computed tomography; GCS: Glasgow Coma Scale; ICH: intracerebral hemorrhage; IQR: interquartile range; MMRS: modified Rankin Disability Scale; MRI: magnetic resonance imaging; ROC: receiver operating characteristic; sE-selectin: soluble endothelial selectin; sICAM-1: soluble intercellular cell adhesion molecule-1; sL-selectin: soluble leukocyte selectin; sP-selectin: soluble platelet selectin; sVCAM-1: soluble vascular cell adhesion molecule-1.

\section{Acknowledgements}

This study was supported by grants from Chang Gung Memorial Hospital Research Project CMRPG880921. None of the authors has any commercial association, such as consultancies, stock ownership, or other equity interests or patent-licensing arrangements. The authors thank Yi-Fang Chiang for her excellent enzyme-linked immunosorbent assay measurement technique.

\section{Author details}

${ }^{1}$ Department of Neurosurgery, Kaohsiung Chang Gung Memorial Hospital, Chang Gung University College of Medicine, 123, Ta-Pei Road, Niao Sung district, Kaohsiung 83304, Taiwan. ${ }^{2}$ Graduate Institute of Medicine, College of Medicine, Kaohsiung Medical University, 100, Shi-Chuan 1st Road, Kaohsiung, 807, Taiwan. ${ }^{3}$ Department of Radiology, Kaohsiung Chang Gung Memorial Hospital, Chang Gung University College of Medicine, 123, Ta-Pei Road, Niao Sung district, Kaohsiung 83304, Taiwan. ${ }^{4}$ Department of Biological Science, 70 Lienhai Road, National Sun Yat-Sen University, Kaohsiung, 80424, Taiwan. ${ }^{5}$ Department of Neurology, Kaohsiung Chang Gung Memorial Hospital, Chang Gung University College of Medicine, 123, Ta-Pei Road, Niao Sung district, Kaohsiung 83304, Taiwan. 'Department of Medicine, Kaohsiung Chang Gung Memorial Hospital, Chang Gung University College of Medicine, 123, Ta-Pei Road, Niao Sung district, Kaohsiung 83304, Taiwan. 7 Department of Emergency Medicine, Kaohsiung Chang Gung Memorial Hospital, Chang Gung University College of Medicine, 123, Ta-Pei Road, Niao Sung district, Kaohsiung 83304, Taiwan. 


\section{Authors' contributions}

$\mathrm{H}-\mathrm{CW}$ and $\mathrm{Y}-\mathrm{JL}$ participated in the design of the study and drafted the manuscript. C-SR, T-HL, B-CC, and C-TK participated in the sequence alignment and clinical evaluation of patients. W-CL interpreted the neuroimaging studies. W-NC and N-WT performed the statistical analysis. C-HL conceived the study, participated in its design and coordination, and helped draft the manuscript. All authors read and approved the final manuscript.

\section{Competing interests}

The authors declare that they have no competing interests.

Received: 27 July 2011 Revised: 8 August 2011

Accepted: 25 November 2011 Published: 25 November 2011

\section{References}

1. Dennis MS, Burn JP, Sandercock PA, Bamford JM, Wade DT, Warlow CP: Long-term survival after first-ever stroke: the Oxfordshire Community Stroke Project. Stroke 1993, 24:796-800.

2. Brown RD, Whisnant JP, Sicks JD, O'Fallon WM, Wiebers DO: Stroke incidence, prevalence, and survival: secular trends in Rochester, Minnesota, through 1989. Stroke 1996, 27:373-380.

3. Mayo NE, Neville D, Kirkland S, Ostbye T, Mustard CA, Reeder B, Joffres M, Brauer G, Levy AR: Hospitalization and case-fatality rates for stroke in Canada from 1982 through 1991. The Canadian Collaborative Study Group of Stroke Hospitalizations. Stroke 1996, 27:1215-1220.

4. Wang J, Dore S: Inflammation after intracerebral hemorrhage. J Cereb Blood Flow Metab 2007, 27:894-908.

5. Aronowski J, Hall CE: New horizons for primary intracerebral hemorrhage treatment: experience from preclinical studies. Neurol Res 2005, 27:268-279.

6. Gong C, Hoff JT, Keep RF: Acute inflammatory reaction following experimental intracerebral hemorrhage in rat. Brain Research 2000, 871:57-65

7. Ma Q, Manaenko A, Khatibi NH, Chen W, Zhang JH, Tang J: Vascular adhesion protein-1 inhibition provides antiinflammatory protection after an intracerebral hemorrhagic stroke in mice. J Cereb Blood Flow Metab 2011, 31:881-893.

8. Yilmaz G, Granger DN: Cell adhesion molecules and ischemic stroke. Neurol Res 2008, 30:783-793.

9. Barone $F C$, Feuerstein $G Z$ : Inflammatory mediators and stroke: new opportunities for novel therapeutics. J Cereb Blood Flow Metab 1999, 19:819-834.

10. Emsley HC, Tyrrell PJ: Inflammation and infection in clinical stroke. J Cereb Blood Flow Metab 2002, 22:1399-1419.

11. Endres M, Laufs U, Merz H, Kaps M: Focal expression of intercellular adhesion molecule-1 in the human carotid bifurcation. Stroke 1997, 28:77-82.

12. Bevilacqua MP: Endothelial-leukocyte adhesion molecules. Annu Rev Immunol 1993, 11:767-804.

13. Springer TA: Leucocyte adhesion to cells. Scand J Immunol 1990 32:211-216.

14. Hafler DA, Weiner HL: MS: a CNS and systemic autoimmune disease. Immunol Today 1989, 10:104-107.

15. Cannella B, Cross AH, Raine CS: Upregulation and coexpression of adhesion molecules correlate with relapsing autoimmune demyelination in the central nervous system. J Exp Med 1990, 172:1521-1524.

16. Kaynar MY, Tanriverdi T, Kafadar AM, Kacira T, Uzun H, Aydin S, Gumustas K, Dirican A, Kuday C: Detection of soluble intercellular adhesion molecule1 and vascular cell adhesion molecule-1 in both cerebrospinal fluid and serum of patients after aneurysmal subarachnoid hemorrhage. $J$ Neurosurg 2004, 101:1030-1036.

17. Kraus J, Oschmann P, Leis S, Neundorfer B, Heckmann JG: High concentrations of SVCAM-1 and SICAM-1 in the cerebrospinal fluid of patients with intracerebral haemorrhage are associated with poor outcome. J Neurol Neurosurg Psychiatry 2002, 73:346-347.

18. Bamford JM, Sandercock PA, Warlow CP, Slattery J: Interobserver agreement for the assessment of handicap in stroke patients [letter]. Stroke 1989, 20:828.

19. Cerebrovascular diseases: prevention, treatment, and rehabilitation Report of a WHO meeting. World Health Organ Tech Rep Ser 1971, 469:1-57.
20. Kraus J, Gerriets T, Leis S, Stolz E, Oschmann P, Heckmann JG: Time course of VCAM-1 and ICAM-1 in CSF in patients with basal ganglia haemorrhage. Acta Neurol Scand 2007, 116:49-55.

21. Wang HC, Lin WC, Yang TM, Lin YJ, Chen WF, Tsai NW, Chang WN, Lu CH: Risk factors for acute symptomatic cerebral infarctions after spontaneous supratentorial intra-cerebral hemorrhage. J Neurology 2009, 256:1281-1287.

22. Armstrong FS, Hayes GJ: Segmental cerebral arterial constriction associated with pheochromocytoma: report of a case with arteriogram. Neurosurg 1961, 18:843-846.

23. Friedman $\mathrm{P}$, Gass $\mathrm{H}$, Magidson M: vasospasm with an unruptured and unoperated aneurysm. Surg Neurol 1983, 19:21-25.

24. Smith RA, Collier HF, Underwood FO: Cerebral vasospasm following myelography. Surg Neurol 1973, 1:87-90

25. White RP, Hagen AA, Morgan H, Dawson WN, Robertson JT: Experimental study on the genesis of vasospasm. Stroke 1975, 6:52-57.

26. Kobayashi H, Hayashi M, Kobayashi S, Kabuto M, Handa Y, Kawano H, Ide H: Cerebral vasospasm and vasoconstriction caused by endothelin. Neurosurgery 1991, 28:673-679.

27. Tsai NW, Lin TK, Chang WN, Jan CR, Huang CR, Chen SD, Cheng KY, Chiang YF, Wang HC, Yang TM, Lin YJ, Lin WC, Chang HW, Lee LH, Lu CH: Statin pre-treatment is associated with lower platelet activity and favorable outcome in patients with acute non-cardio-embolic ischemic stroke. Crit Care 2011, 15:R163.

28. Leker RR, Khoury ST, Rafaeli G, Shwartz R, Eichel R, Tanne D, NASIS Investigators: Prior use of statins improves outcome in patients with intracerebral hemorrhage: prospective data from the National Acute Stroke Israeli Surveys (NASIS). Stroke 2009, 40:2581-2584.

29. White HD, Simes RJ, Anderson NE, Hankey GJ, Watson JD, Hunt D, Colquhoun DM, Glasziou P, MacMahon S, Kirby AC, West MJ, Tonkin AM: Pravastatin therapy and the risk of stroke. N Engl I Med 2000, 343:317-326

30. Qureshi Al, Ling GS, Khan J, Suri MF, Miskolczi L, Guterman LR, Hopkins LN: Quantitative analysis of injured, necrotic, and apoptotic cells in a new experimental model of intracerebral hemorrhage. Crit Care Med 2001, 29:152-157.

31. Kwak BR, Mach F: Statins inhibit leukocyte recruitment: new evidence for their anti-inflammatory properties. Arterioscler Thromb Vasc Biol 2001, 21:1256-1258.

32. Veillard NR, Mach F: Statins: the new aspirin? Cell Mol Life Sci 2002, 59:1771-1786.

doi:10.1186/cc10568

Cite this article as: Wang et al:: The association between serum adhesion molecules and outcome in acute spontaneous intracerebral hemorrhage. Critical Care 2011 15:R284.

\section{Submit your next manuscript to BioMed Central and take full advantage of:}

- Convenient online submission

- Thorough peer review

- No space constraints or color figure charges

- Immediate publication on acceptance

- Inclusion in PubMed, CAS, Scopus and Google Scholar

- Research which is freely available for redistribution
C Biomed Central 